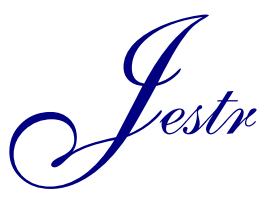

\title{
Direction Estimation using Patterns of User Movement
}

\author{
Nagham A. Al-Tarawneh ${ }^{1, *}$, Ahmad H. Abadleh ${ }^{1}$ and Zaid T. Alhalhouli ${ }^{2}$ \\ ${ }^{I}$ Department Of Computer Science, Mutah University, Jordan \\ ${ }^{2}$ Department of Computer and Information Technology, Tafila Technical University, Jordan
}

Received 3 March 2018; Accepted 12 July 2019

\begin{abstract}
This study mainly aims to find accurate directions in indoor environments by combining smartphone acceleration readings with WiFi received signal strength (RSS) readings. Patterns of user movement serve as the basis of this approach. These patterns are effectively utilized to detect user direction on the basis of the correlation between the RSS and acceleration values. A series of experiments was conducted to test the proposed approach. The proposed approach achieves $95 \%$ accuracy and thus shows great potential as a practical solution for estimating directions in indoor environments.
\end{abstract}

Keywords: acceleration, received signal strength, line of sight, non-line of sight, direction.

\section{Introduction}

Positioning systems have attracted increasing interest in recent years because of the need for position information at any given time and place. Positioning systems may be categorized as indoor and outdoor positioning systems [1]. For outdoor localization, global positioning system (GPS) is the most popular positioning system available. GPS is a 24satellite system that offers global coverage and accuracy within 1-5 $\mathrm{m}$ [2] in providing information about the locations of objects or people on the Earth's surface. However, GPS is not always accurate in indoor environments because of insufficient line-of-sight (LOS) transmission channels between satellites and indoor receivers [3]. Indoor positioning (IP) systems are useful in locating objects, people, and animals within buildings.

Indoor positioning is generally realized with wireless fidelity (WiFi). Received signal strength (RSS) fingerprints may be obtained from most commercial wireless network equipment, such as WiFi devices [4]. Smartphones, in particular, have become popular because of the convenience they provide. Modern smartphones have multiple sensors, which can be combined to ensure precise indoor positioning [5]. These internal sensors detect user-phone proximity for user activity recognition, indoor tracking, or indoor localization [6].

Today, more than $80 \%$ information are concerning to spatial location, and people spend about $80 \%-90 \%$ time of their entire life indoors [7]. With the generalization of information and communication technology, people are urgently demanding accurate indoor location information. Such information is especially crucial in public places such as office buildings, prisons, and underground parking spaces. In such environments that primarily involve

\footnotetext{
*E-mail address: zaid_halhouli@yahoo.com

ISSN: $1791-2377$ @2019 School of Science, IHU. All rights reserved.

doi:10.25103/iestr.124.24
}

pedestrian navigation, GPS is not a reliable tool.

The main motive behind this research lies in finding the accurate dire ction of the user in indoor environments. Many researches depended on the digital compass in direction estimation, but the digital compass sensor is heavily affected by magnetic field caused by the proximity of artificial magnetic field or large ferrous structures [8]. Hence, this effect leads to inaccuracy in detecting the direction. Practically, this makes the compass unreliable for direction determination in indoor environments. In the current work, the use of WiFi RSS and smartphone sensors is proposed to obtain accurate directions in indoor environments. The proposed approach identifies user movement (walking, slow walking, and standing) by using an accelerometer sensor installed on smartphones. The identification is realized when smartphones are held by users. The data derived from this accelerometer sensor are then analyzed. Accurate directions are obtained by the combination of the WiFi RSS reading and the accelerometer data.

A new method for direction estimation based on the patterns of user movement is proposed in this work. The patterns of user llmovement are converted into states that represent movement options. In the proposed approach, RSS and acceleration values are utilized to detect movement states, which aid in the detection of user directions.

\section{Related Works}

Numerous works that promote wireless technologies, such as Bluetooth and WiFi, have been conducted because of the increasing recognition of the value of indoor positioning. He et al. [9] proposed a WiFi iLocate system for tracking smartphones in indoor environments. In this system, a Gaussian process regression trains the collected WiFi RSS datasets, and a particle filter estimates smartphone location. Similar to other WiFi-based fingerprints, this schema builds discrete location fingerprints during offline training and 
Nagham A. Al-Tarawneh, Ahmad H. Abadleh and Zaid T. Alhalhouli/

Journal of Engineering Science and Technology Review 12 (4) (2019) 195 - 201

estimates each location using Bayesian decision rules with the recognized signal strength. The collected fingerprints are interpolated by a Gaussian process and also the location of the Smartphone is specified by a particle filter.

$\mathrm{Wu}$ et al. in 2016 [10] presented a WiDir system thatcan estimate moving directions using WiFi channel state information (CSI) information without the need for any device. Human motion changes multipath distributions and the WiFi CSI at the recipient end. Thus, WiDir analyzes the phase change dynamics from various WiFi subcarriers according to the Fresnel zone model and then derives the walking direction. This WiDir system is capable of estimating human walking directions with an average error of less than $10^{\circ}$.

\subsection{Indoor Positioning System Techniques}

Indoor positioning techniques are categorized as triangulation, scene analysis, and proximity techniques [11]. In triangulation, the location of a target place is estimated on the basis of the geometric properties of triangles [12]. This indoor positioning technique involves lateration and angulation [13]. Lateration estimates the position of targets according to their distance from multiple reference points; the three main lateration methods are time of arrival (TOA) [13], time difference of arrival (TDOA) [14], and RSS [13]. Angulation locates targets by calculating angles relative to multiple reference points; it mainly employs angle of arrival (AOA) [11].

Fingerprinting, which is also known as scene analysis, is a widely employed estimation technique. It not only achieves accurate results but also eliminates the need for the location of access points (APs) to make an estimation. Moreover, localization is still possible even with the APS disabled for a certain period [14].

Finally, the proximity technique combines targets with the nearest antennas or reference points. Positioning based on proximity requries the detection of the presence of a device within a certain range [15].

Direction Estimation

Various methods based on sensors, such as compasses, accelerometers, and orientation sensors, are used to detect the directions of user movement. Smartphones today are equipped with digital compasses, which are mainly utilized to determine orientation. Roy et al. [16] proposed a walkcompass that detects walking direction by exploiting smartphone sensors and ignoring the orientation of the phones on the body. The study also analyzed the relationship between human walking and its impact on phone condition, along with the method for estimating and eliminating magnetic interference from compass data.

Kusber et al. [17] proposed a method for detecting the orientation and movement direction of users who carry their smartphones inside their trouser pockets. Smartphone orientation and movement direction alignment (SOMDA) initially detects the orientation of the smartphone top using a compass and an orientation sensor. Then, SOMDA detects the orientation of the smartphone screen and the direction of user movement by observing the compass and the accelerometer during actual user movement. The accuracy of the results can reach $96 \%$.

A gyroscope is known to employ up to three axes. The spatial orientation of a solid object is based on pitch and rotation around the $\mathrm{x}$-axis, roll and rotation around the $\mathrm{y}$ axis, and azimuth rotation around the z-axis [18]. A gyroscope is a highly sensitive device the can detect spin movement. Similar to accelerometers, gyroscopes return three-dimensional values. These devices also close the gap when compasses are disturbed and thus improve system reliability. Barthold et al. [19] exploited the built-in gyroscope in the Nexus $\mathrm{S}$ smartphone to addressthe interference problems of orientation sensors.

A gyroscope sensor is utilized to examine the level of precision that is possible in indoor navigation. The use of this device involves an inertial navigation system (INS) that can support users in navigating unknown indoor environments through the continuous calculation of their positions and motions. The solution depends on the integrated use of position sensors and movement. Since, the applications that based on the use of measures coming from orientation sensors, such as digital compasses and accelerometers, are affected by external magnetic interferences resulting in inaccurate direction information. The problem is resolved with the use of gyroscope for determining orientation. In this regard, gyroscopes provide better orientation information than digital compasses [18].

Zhou et al. [20] proposed the use of inertial sensors to improve the dead reckoning algorithm and help firefighters in perceiving their locations on site when putting outfires. Accelerometers and gyroscopes were utilized to determine the step, stride, and heading, all of which make up user motion. An accelerometer was used in the study to count steps, and a gyroscope was utilized to determine any changes in direction.

\subsection{Limitations of Direction Estimation}

Integrated digital compasses in smartphones are mainly used in most mobile navigation systems. However, their measurements are prone to large errors due to the presence of metallic objects, electronic devices, and magnetic fields that tend to adversely affect system accuracy and reliability [18]. King et al. in 2006 [21] also indicated that digital compass errors could reach $23^{\circ}$.

LifeMap is a smartphone-based context provider [22]. In particular, LifeMap can provide mobile users with advanced location-based services. LifeMap uses inertial sensors to overcome the limitations of GPS and provide indoor location information. It then generates user context by combining indoor location information with GPS and WiFi positioning systems. LifeMap detects user direction with a digital compass. However, magnetic fields were found to affect digital compasses.

\section{Proposed Approach}

\subsection{Overview}

Fig. 1 shows the overall system architecture. The proposed approach consists of three phases, namely, the movement state, the direction algorithm phase, and the check movement state.

\subsection{System Architecture}

Fig.1 illustrates the overall representation of the proposed approach. Data collection is achieved with a smartphone accelerometer sensor and the RSS emitted by APs that cover a given building. A direction algorithm is then implemented to combine the RSS and acceleration readings. Subsequently, movement is classified into three categories according to walking speed. Finally, the system identifies movement direction by detecting the movement state and the correlation among the RSS values. This process is based on the assumption that the entire building is covered by APs. 
Nagham A. Al-Tarawneh, Ahmad H. Abadleh and Zaid T. Alhalhouli/

Journal of Engineering Science and Technology Review 12 (4) (2019) 195 - 201

This assumption is reasonable because most public buildings provide WiFi services to users.

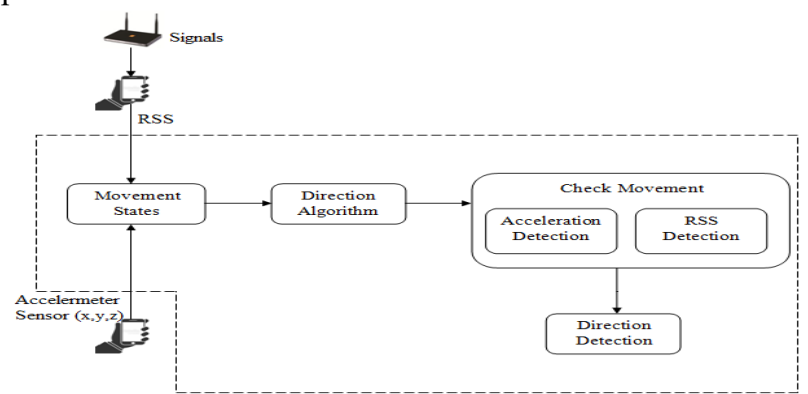

Fig. 1. Block diagram of the proposed approach.

\subsubsection{Movement State}

Fig. 2 displays the finite state machine for detecting the state of movement. The figure explains how acceleration and RSS are affected when a user moves from one activity to another. The transition between states is explained as follows.

- Slow walking to regular walking: acceleration increases, and the RSS values are consistent.

- Regular walking to slow walking: acceleration decreases, and the RSS values are consistent.

- Regular walking to stopping: acceleration approaches zero, and the RSS values are consistent.

- Stopping to regular walking: acceleration increases, and the RSS values are consistent.

- Slow walking to stopping: acceleration approaches zero, and the RSS values are consistent.

- Stopping to slow walking: acceleration increases, and the RSS values are consistent.

- Slow walking to turning left/right: acceleration changes, and the RSS values change.

- Stopping to turning back: acceleration changes, and the RSS values change significantly.

- Stopping to turning left/right: acceleration changes, and the RSS values change slightly.

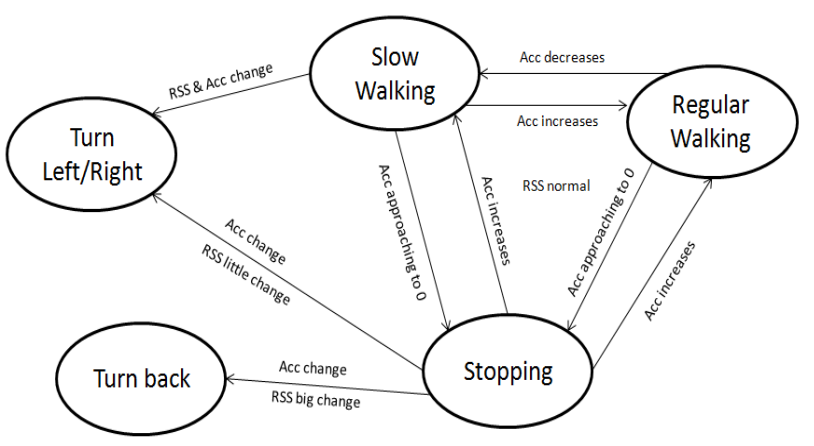

Fig. 2. Transition state diagram between activities.

\subsubsection{Direction Algorithm Phase}

We observed that changes in acceleration values imply changes in direction. State movement can be in the forms of normal walking, slow walking, and standing according to changes in acceleration values. Changes in RSS values are interpreted as follows. A significant change in RSS values indicates that the user is turning back, a slight change indicates that the user is turning left or right, and a consistent change indicates that the direction remains the same.

Algorithm1 describes the steps to determine movement direction; the three-axis accelerometer readings and RSS readings serve as the inputs.
Alg. 1: Overall architecture for direction detection.

Input: Acceleration(x, y, z), RSS.

Output: detection direction.

1. Check_Acc (x, y, z)

2. If (move $=$ 'stop') then

3. RSS_Result= Check_RSS (RSS_value)

4. If (RSS_Result is little change for time window) then

5. Check_Direction (RSS, Acc) //Direction is turn left/right

6. End if

7. If (RSS_Result is big change for time window) then

8. Direction= Opposite Direction

9. $\quad$ End if

10. If (RSS_Result is consistent) then

11. Direction= Same Direction

12. End if

13. End if

14. If (move= 'slow walking') then

15. RSS_Result= Check_RSS (RSS_value)

16. If (RSS_Result is little change for time window) then

17.

is turn left/right

18. End if

19. If (RSS_Result is consistent) then

20. Direction= Same Direction

21. End if

22. End if

23. If (move $=$ 'normal walking') then

24. Direction= Same Direction

25. End if

Algorithm1 works as follows. First, it detects movement states according to the RSS and acceleration values. Second, it detects movement direction according to the detected movement states and the acceleration and RSS values. Consider a case characterized by the detection of a sudden and significant difference in RSS values and the occurrence of changes in acceleration from the stopping state into the walking state; the direction here is most likely the opposite of the current one. Under this mechanism, the user's body blocks RSS signals while walking, and RSS values increase when the user turns back because this user's body no longer blocks the signals.

\subsubsection{Check Movement State}

Movement states are determined according to the acceleration and RSS values.

Acceleration Detection

An accelerometer is a sensor for measuring acceleration $\left(\mathrm{m} / \mathrm{s}^{2}\right)$ along one or more axes.

The proposed approach collects acceleration values as follows. First, acceleration readings are collected for all cases involving users walking (regular walking, slow walking, stopping, turning back, and turning left/right). The collected data are divided into parts by a time window. Second, the magnitude value for each reading in each part is calculated using Equation 1. Third, a simple high pass filter is applied to the magnitude of the acceleration values to remove the Earth's gravity through Equation 2. Subsequently, the average magnitude for all values is computed. Finally, the standard deviation (StD) of the magnitude values is computed. 
Nagham A. Al-Tarawneh, Ahmad H. Abadleh and Zaid T. Alhalhouli/

Journal of Engineering Science and Technology Review 12 (4) (2019) 195 - 201

Magnitude $=\sqrt{X^{2}+Y^{2}+Z^{2}}$

$X$ is the acceleration force along the $x$-axis (including gravity), $\mathrm{m} / \mathrm{s}^{2}$.

$Y$ is the acceleration force along the $y$-axis (including gravity), $\mathrm{m} / \mathrm{s}^{2}$.

$Z$ is the acceleration force along the $z$-axis (including gravity), $\mathrm{m} / \mathrm{s}^{2}$.

$\mathrm{Acc}=|\mathrm{Mag}-G|$

Mag is the magnitude value for each reading in each time window.

$G$ is the gravity force.

The acceleration threshold is set on the basis of the StD value. Therefore, for each movement state, the threshold is set in the same way. For the turning back stage for instance, the RSS threshold is higher than that for turning left/right.

RSS Detection

According to the propagation model of WiFi signals, signal intensity decreases with an increase in propagation distance. As the RSS level changes, the average value must be used.

Figs. 3 and 4 present the LOS and non-line of sight (NLOS), respectively. In LOS deployment, APs and mobile stations may not suffer from any obstruction. LOS deployment provides better signal strength and higher throughput than NLOS deployment. In NLOS communication, obstacles may come between the transmitter and the receiver. In such a case, the signal reaches the receiver only after going through obstructions. For example, a person's body is in the same direction as a signal.

These features of LOS and NLOS are used in the proposed approach to detect user movement states. The RSS values form the transition values from one state to another. Direction detection is facilitated by the combined changes of RSS and acceleration values.
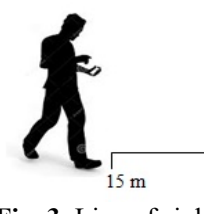

Fig. 3. Line of sight.

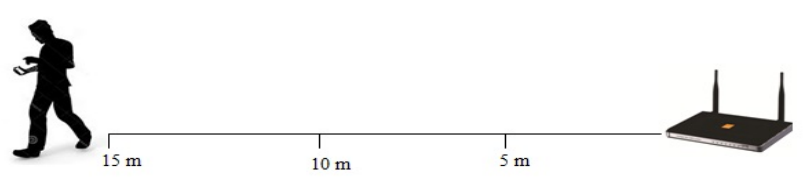

Fig. 4. Non-line of sight.

As shown in the LOS case in Fig.3, no obstacle exists between the user and the AP. Consequently, the signal reaches the user clearly. By contrast, the NLOS case illustrated in Fig. 4 shows that the user's body blocks the signal between the mobile station and the AP.

\section{Evaluation}

\subsection{Experiment Setup}

A series of experiments were conducted at the second floor of the Faculty of Information Technology building in Mutah University. Fig.5 presents the test environment. The experiment environment involved four APs serving as reference nodes that can be easily sensed by users' mobile phones.

The Faculty of Information Technology office measures $51.6 \mathrm{~m}$ in length and $44.4 \mathrm{~m}$ in width, makes up an area of $2291.04 \mathrm{~m}^{2}$, and includes more than 25 sections.

The model of APs used in our experiments is ZXHN H108N, which is an asymmetric digital subscriber line (ADSL) access device that supports multiple line modes for bridge head servers. Each AP supports ADSL2/ADSL2+, is backward compatible to ADSL, and supports a wide input voltage range of $100-240 \mathrm{~V}$. According to the settings of the central office digital subscriber line access multiplexer, the AP provides an auto negotiation capability for different standards.

This AP has satisfactory features and provides a broadband Internet connection through a high-speed ADSL connection, secure wireless encryption modes, and a firewall for network security.

All experiments were conducted using an Android-based Galaxy GRAND 2 smartphone, which is equipped with accelerometer sensors and WiFi. These experiments were conducted during the day when RSS might be affected by obstacles such as pedestrians between public APs and a user's smartphone used to collect RSS information. Each of the APs and the smartphone were supported by IEEE 802.11 $\mathrm{b} / \mathrm{g} / \mathrm{n} \mathrm{WiFi}$ interfaces.

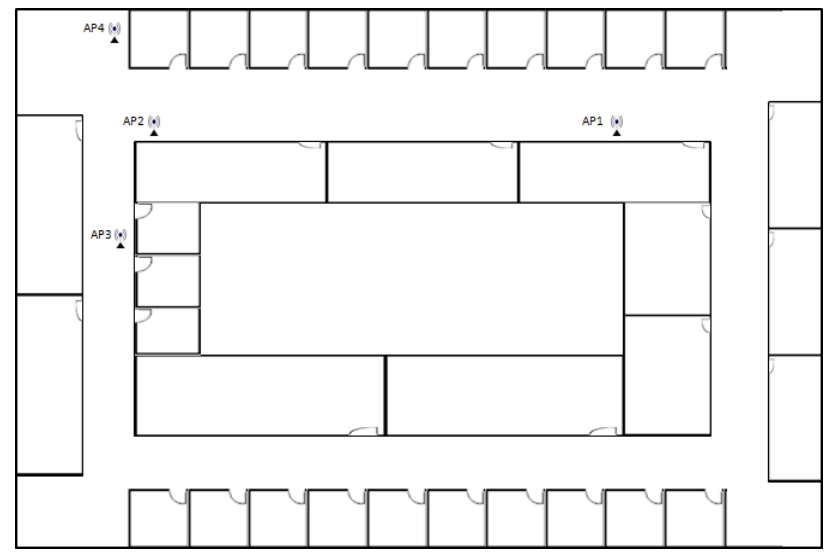

Fig. 5.Test environment

During each experiment, a user holds the smartphone to collect data. Data were collected with two software applications installed on the smartphone. The first application, the Accelerometer Meter, was used to obtain acceleration data. The second application, WiFi Analyzer, was used to capture signal strength.

\subsection{Experiment Result}

\subsubsection{Acceleration analysis}

The acceleration data were analyzed with three experiment scenarios.

\section{A. Scenario 1}

The user walks straight from AP1 to AP2. The changes in acceleration when this user walks in different walking styles are examined.

Accelerometer data are collected when the user walks straight. Fig. 6 shows the significant changes in acceleration values when moving from one state to another. 
Nagham A. Al-Tarawneh, Ahmad H. Abadleh and Zaid T. Alhalhouli/

Journal of Engineering Science and Technology Review 12 (4) (2019) 195 - 201

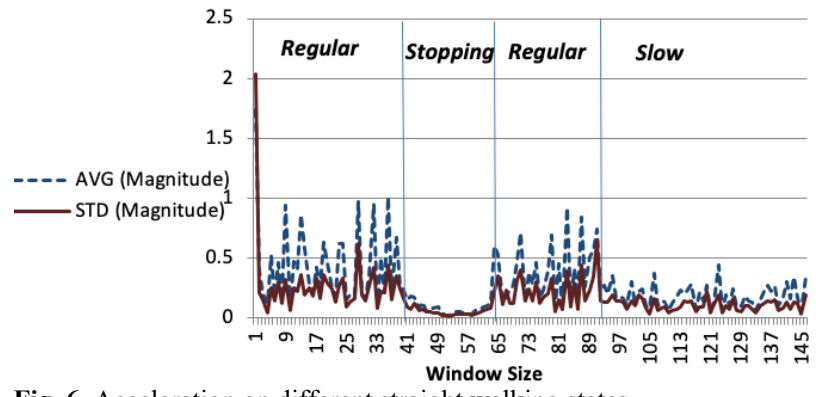

Fig. 6. Acceleration on different straight walking states.

\section{B. Scenario 2}

In this scenario, the differences in acceleration when the user turns back while walking are analyzed.

Fig. 7 shows the results of this scenario. A considerable change was observed in the values when turning back while walking as the user passes through the stopping or walking state.

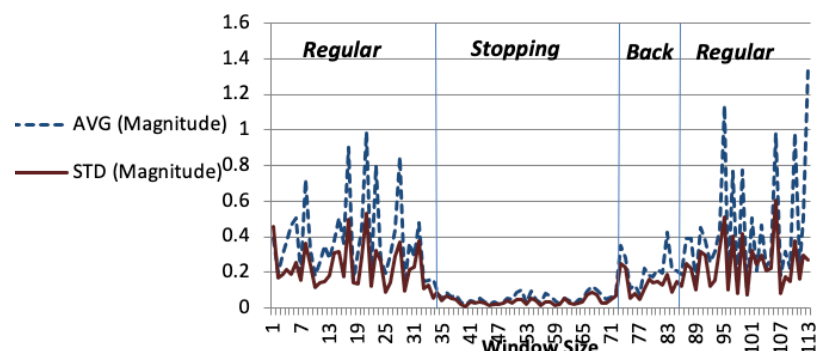

Fig. 7.Acceleration when user turns back while walking straight. represents stopping, then turn back. (

\section{Scenario 3}

In this scenario, the user walks then turn to the right.

Fig. (8.a) shows when the user walks regularly and then moves to the right. We note that the average and the standard deviation of acceleration values begin to decrease when moving from regular walking to the right as shown from window 37 towindow 47. After that, the values begin to increase when moving to regular walking.But we conclude from Fig. (8.b) that the average and the standard deviation of acceleration values increase when moving from slow walking to the right. Acceleration values increase significantly when moving to regular walking.

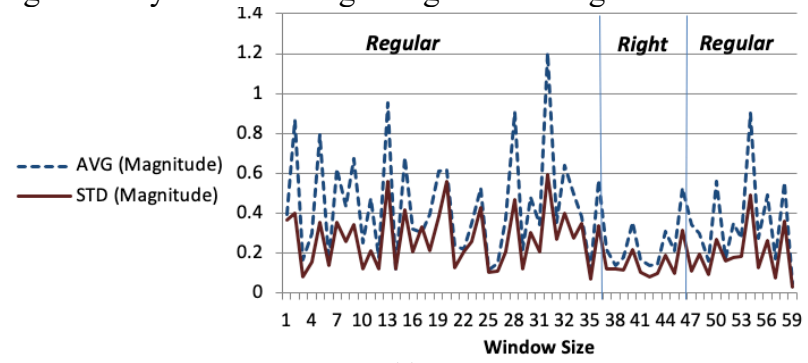

(a)

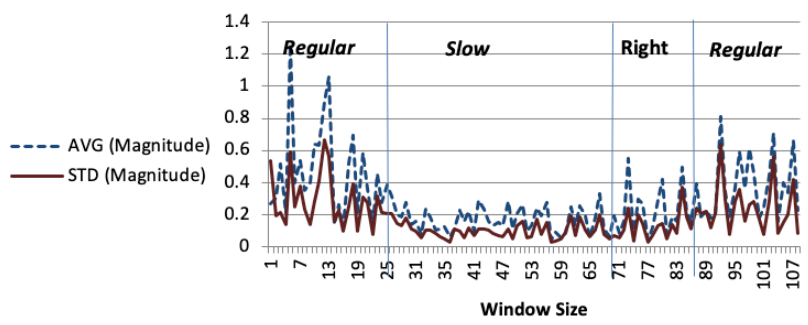

(b)
Fig. 8. Acceleration when user turns right while walking straight. (a) represents walking regularly,then turn right. (b)representswalking slowly, then turn right.

\subsubsection{RSS analysis}

This subsection presents the analysis of the RSS values and the correlation between WiFi APs for direction detection.

\section{A. Scenario 1}

The user starts walking from AP1 toward AP2. Fig.9 shows the results of this scenario.

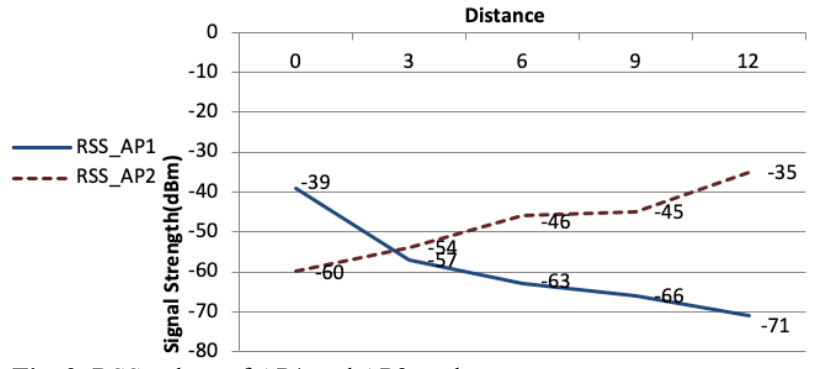

Fig. 9. RSS values of AP1 and AP2 as the user moves.

As shown in Fig.9, the RSS values of AP1 start strong and then weaken as the user walks, and the RSS values of AP2 start weak and then strengthen. Specifically, the RSS values of AP1 move a way from the LOS as the user's body blocks the signal, while the RSS values of AP2 becomes increasingly strong because the user faces AP2 while walking, which means that the signal is in LOS.

\section{B. Scenario 2}

We examined the changes in the RSS values when the user turns back during movement. The results of our analysis are shown in Fig. 11.

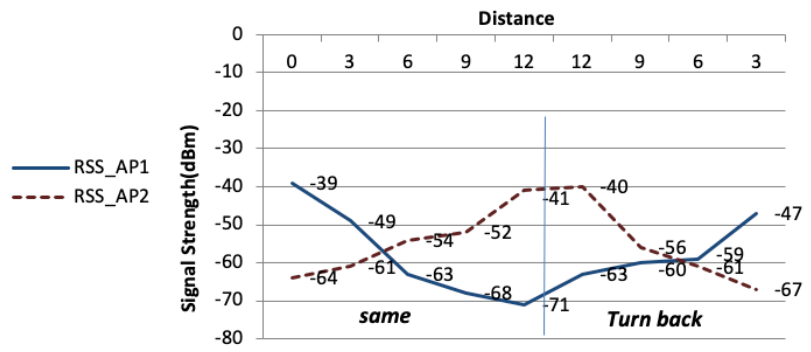

Fig. 10. RSS values for AP1 and AP2 when turning back.

Fig. 10 proves that RSS values become the opposite when the user turns back. Obviously, the RSS values of AP1 weaken as the user walks toward AP2 and then strengthen when the user turns back as the signals enter the LOS.

\section{Scenario 3}

We studied the changes and the correlation between RSS values from different APs. Figs. 11 and 12 show the results.

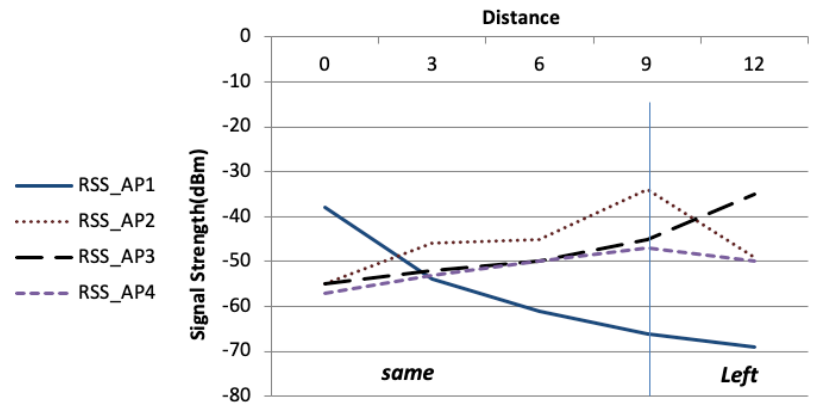

Fig. 11. RSS values when user turns left. 
Nagham A. Al-Tarawneh, Ahmad H. Abadleh and Zaid T. Alhalhouli/

Journal of Engineering Science and Technology Review 12 (4) (2019) 195 - 201

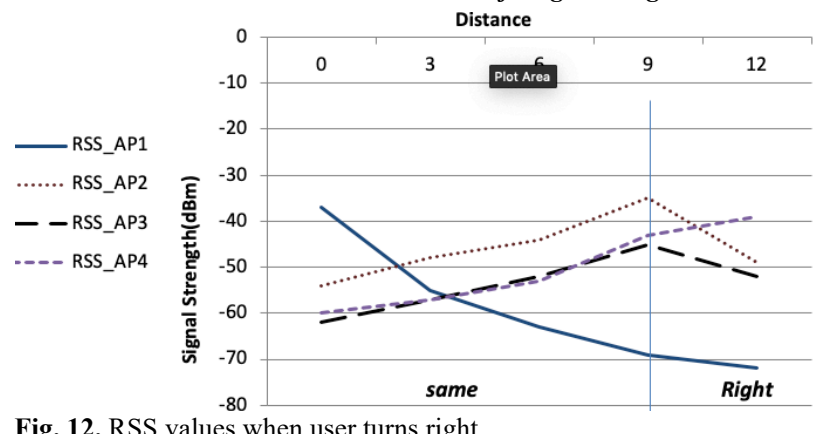

Fig. 12. RSS values when user turns right.

Figs. 11 and 12 illustrate the changes in the RSS values of the APs. The figures show four APs distributed at different distances. We infer that the RSS values for AP3 strengthen when the user turns left; in this case, the user faces AP3. By contrast, the RSS values of AP3 weaken and the RSS values of AP4 strengthen; hence, the user faces AP4.

Therefore, the system detects direction by checking user movement. The acceleration values and the correlation between the RSS values from different APs ultimately determine where the user is heading in an indoor environment.

\subsection{Error Analysis}

Accuracy in this work refers to the ability of the proposed approach to achieve minimal error rates in direction estimation. The error rate is calculated using Eq. 3.

Error rate $=(E r 1+E r 2+\cdots+E r n) / n$

Er is the error for each window.

$n$ is the total number of samples.

With the empirical threshold for each movement known, the error rate is calculated according to the values that fail to meet the empirical threshold condition for all movement states. The total error rate is calculated by Eq. 3. The accuracy of the system in detecting state and direction is approximately $95 \%$.

In linear regression, the correlation between the RSS values and distance is modeled by fitting a linear equation to the observed data. The regression line is calculated by Eq.4.

$\mathrm{Y}=m \times d+c$

$m$ is the slope of the line.

$d$ is the distance along which the values are calculated. $c$ is aconstant.

Fig. 13 illustrates the turn right scenario in which we noted that the regression line equation and R-squared values are different for each AP.

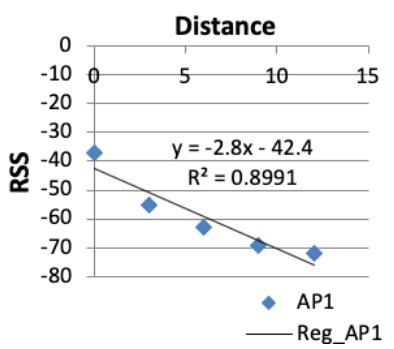

(a)

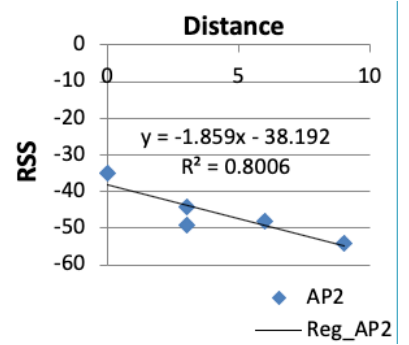

(b)

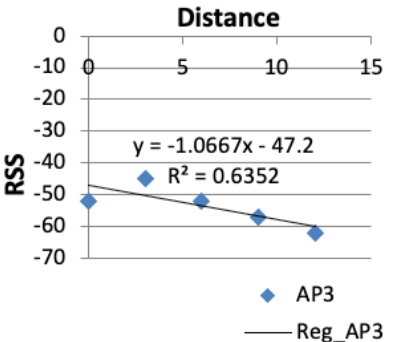

(c)

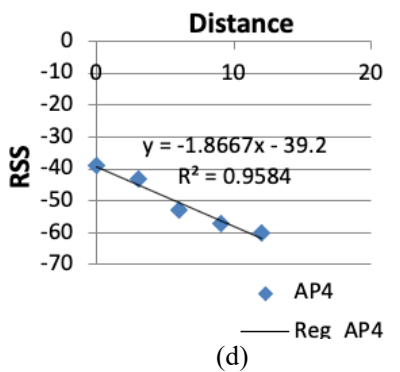

Fig.13.Turn right: linear regression plot for RSS values of different APs.a) Linear regression for AP1.b) Linear regression for AP2. c)Linear regression for AP3. d)Linear regression for AP4.

$\mathrm{R}$-squared is a statistical measure of how close the data are to the fitted regression line. As shown in Fig.14, the Rsquared value of AP4 is the highest; hence, the user moves to the right. The results are summarized as follows.

- Positive correlation:

The high R-squared value of AP1 is associated with the high $\mathrm{R}$-squared value of AP4.

- Negative correlation:

The high R-squared value of AP1 is associated with the low $\mathrm{R}$-squared values of AP2 and AP3.

The turn left scenario is shown in Fig .15. Each graph (a, b, $\mathrm{c}, \mathrm{d})$ shows the regression line equation and R-squared values.

\section{Comparison}

The main difference between our approach and the existing ones is that we provide the direction without the need of digital compass which most of the existing approach rely on. Our approach depends on the movement pattern of the users instead of using the inaccurate digital compass to detect the directions. Moreover, to the best of our knowlodge, our approach is the first that taken into account the relation between the RSS values and the movement style of the users to detect the direction.

\section{Conclusion}

The research proposes a novel direction estimation approach. The proposed system utilizes sensor data such as accelerometer sensor and Wi-Fi Received Signal Strength to determine accurate direction. The proposed approach for direction estimation requires smartphone to be carried by hand. In the first stage, the acceleration values are collected from a 3-axis accelerometer. In each part, the magnitude for each reading is calculated. A filter is then applied to the magnitude values to eliminate gravity forces. Finally, the standard deviation and the average of all values are calculated after a filter operation. Following the analysis of acceleration data, the empirical thresholds are determined along with the movement states. RSS readings are subsequently collected from the distributed APs in the building to determine user direction according to the correlation among the RSS values. Finally, the accelerometer data are combined with the RSS readings to determine accurate directions.

The proposed system satisfies the following objectives of our research. The first is to determine movement states by analyzing acceleration values. The second is to determine directions on the basis of user movement state. The last 
Nagham A. Al-Tarawneh, Ahmad H. Abadleh and Zaid T. Alhalhouli/

Journal of Engineering Science and Technology Review 12 (4) (2019) 195 - 201

objective is to exploit the RSS values in distinguishing accurate directions.

Several experiments were performed to prove the efficiency of the proposed approach. For the movement states, the overall accuracy was $95 \%$, and the error rate was $5 \%$. Our test results indicated that the proposed system can achieve better accuracy than other approaches. System expansion should be considered to support different ways to carry smartphones, a part from using one's hand.

This is an Open Access article distributed under the terms of the Creative Commons Attribution License

\section{References}

[1] A. Alarifi, A. Al-Salman, M. Alsaleh, A. Alnafessah, S. AlHadhrami, M. A. Al-Ammar and H. S. Al-Khalifa, "Ultra Wideband Indoor Positioning Technologies: Analysis and Recent Advances," Sensors 16.5, p. 707, 2016.

[2] J. Liu, "Survey of Wireless Based Indoor Localization Technologies," http://www.cse.wustl.edu/ jain/cse57414/ftp/indoor.pdf, Washington, 2014.

[3] H. Zou, B. Huang, X. Lu, H. Jiang and L. Xie, "A Robust Indoor Positioning System Based," IEEE TRANSACTIONS ON WIRELESS COMMUNICATIONS, vol. 15, pp. 1252-1266, FEBRUARY 2016.

[4] A. Murugan, R. and R. , "Localization based User Tracking Using RSSI," in International Conference on Innovations in Engineering and Technology (ICIET'16), India, 2016.

[5] Y. Liu, M. Dashti, M. A. Abd Rahman and J. Zhang, "Indoor Localization using smartphone inertial sensors," IEEE, pp. 1-6, 2014.

[6] S. Mazilu and G. Tröster, "A Study on Using Ambient Sensors from Smartphones for Indoor Location Detection," IEEE, pp. 1-6, 2015.

[7] X. Zhu and Y. Feng, "RSSI-based Algorithm for Indoor Localization," Communications and Network, pp. 37-42, 2013.

[8] F. H. Teixeira Pinto, "An Indoor Localization Solution for Mobile Devices," FACULDADE DE ENGENHARIA DA UNIVERSIDADE DO PORTO: Master's thesis, 2011.

[9] X. He, S. Badiei, D. Aloi and J. Li, "WiFi iLocate: WiFi based Indoor Localization for "Smartphone," Wireless Telecommunications Symposium (WTS), 2014. IEEE, pp. 1-7, 9 April 2014.

[10] D. Wu, D. Zhang, C. Xu, Y. Wang and H. Wang, "WiDir: Walking Direction Estimation Using Wireless Signals," In Proceedings of the 2016 ACM International Joint Conference on Pervasive and Ubiquitous Computing, pp. 351-362, 2016.

[11] Z. Farid, R. Nordin and M. Ismail, "Recent Advances in Wireless Indoor Localization Techniques," Journal of Computer Networks and Communications, pp. 1-13, 2013.

[12] J. J. Khalifeh, Z. M. Kassas and S. S. Saab, "Indoor Localization Based on Floor Plans and Power Maps:," In Proceedings of ION GNSS Conference, pp. 2291-2300, September 2015.
[13] H. Liu, H. Darabi, P. Banerjee and J. Liu, "Survey of Wireless Indoor Positioning Techniques and Systems," IEEE Transactions on Systems, Man, and Cybernetics, Part C (Applications and Reviews), pp. 1067-1080, 2007.

[14] G. Kul, T. Özyer and B. Tavli, "IEEE 802.11 WLAN based Real Time Indoor Positioning: Literature Survey and Experimental Investigations," Procedia Computer Science 34, pp. 157-164, 2014.

[15] A. M. Abusara, "Indoor Positioning Techniques And Approaches For Wi-Fi Based Systems," United Arab Emirates: Faculty of the American University of Sharjah, 2015.

[16] N. Roy, H. Wang and R. R. Choudhury, "I am a Smartphone and I can Tell my User's Walking Direction," Proceedings of the 12th annual international conference on Mobile systems, applications, and services. ACM, pp. 329-342, 2014.

[17] R. Kusber, A. Q. Memon and D. Krol, "Direction Detection of Users Independent of Smartphone Orientations," In Vehicular Technology Conference (VTC Fall), 2015 IEEE 82nd, pp. 1-6, 2015.

[18] V. Marotto, A. Serra, D. Carboni, M. Sole, T. Dessi and A. Manchinu, "Orientation Analysis through a Gyroscope Sensor for Indoor Navigation Systems," In Proc. 4th Int. Conf. Sensor Device Technol. Appl., pp. 85-90, 2013.

[19] C. Barthold, K. P. Subbu and R. Dantu, "Evaluation of gyroscopeembedded mobile phones," In Systems, Man, and Cybernetics (SMC), 2011 IEEE International Conference, pp. 1632-1638, 2011.

[20] Z. Zhou, T. Chen and L. Xu, "An Improved Dead Reckoning Algorithm for Indoor Positioning Based on Inertial Sensors," International Conference of Electrical, Automation and Mechanical Engineering, pp. 369-371, 2015.

[21] T. King, S. Kopf, T. Haenselmann, C. Lubberger and W. Effelsberg, "COMPASS: A Probabilistic Indoor Positioning System Based on 802.11 and Digital Compasses," Proceedings of the 1st international workshop on Wireless network testbeds, experimental evaluation \& characterization. ACM, pp. 34-40, 2006.

[22] Y. Chon and H. Cha, "LifeMap: Smartphone-based context provider for location-based services," IEEE Pervasive Computing 10, pp. 58-67, 2011. 